\title{
Childhood Small Intestinal Carcinoma
}

National Cancer Institute

\section{Source}

National Cancer Institute. Childhood Small Intestinal Carcinoma. NCI Thesaurus. Code C123933.

A small intestinal carcinoma occurring in childhood. 